\title{
Ehrlichia Meningoencephalitis: A Case Series
}

\author{
Morena J*, Antimisiaris M and Singh D \\ Department of Neurology, The Ohio State University, \\ USA
}

*Corresponding author: J onathan Morena, 395 W 12th Ave \#7, Columbus, OH 43210, USA

Received: January 08, 2021; Accepted: February 27, 2021; Published: March 06, 2021

\section{Abstract}

Objective: We present the distinct clinical and laboratory findings in two cases of Ehrlichia meningoencephalitis, along with one suspected case.

Background: The number of cases of Ehrlichia chaffeensis reported to the CDC has more than doubled from 2007-2017. A PubMed literature search using the words "Ehrlichiosis and meningoencephalitis" revealed five case reports with neurologic manifestations.

Design/Methods: This is a retrospective observational study. Two elderly patients presented with encephalopathy, fever, transaminitis, thrombocytopenia, a positive $E$. chaffeensis Polymerase Chain Reaction (PCR) in Serum, and Cerebrospinal Fluid (CSF) with a lymphocytic or neutrophilic pleocytosis and elevated protein. One patient had similar symptoms and a positive $E$. chaffeensis PCR, but lumbar puncture was unable to be performed due to severe thrombocytopenia. They presented in May or June. Doxycycline was started within 2-3 days after presentation to the hospital. Follow up five months later revealed all patients were close, or back to baseline.

Results and Conclusions: Suspicion of Ehrlichia meningoencephalitis should be raised in elderly patients presenting with fever and encephalopathy in the summer season with history of tick bite or residence in wooded areas. Thrombocytopenia and transaminitis should raise further suspicion. CSF studies typically show a lymphocytic pleocytosis and elevated protein. PCR technique allows for direct detection of pathogen-specific DNA and is the preferred method of detection during the acute phase of illness. Prompt treatment with doxycycline results in good outcomes. Doxycycline is not included in the typical meningitis regimen, therefore, this disease is important to quickly identify as delay in Doxycycline can result in worse outcomes.

Keywords: Meningitis; Encephalitis; Meningoencephalitis; Encephalopathy; Ehrlichiosis

\section{Introduction}

The number of cases of Ehrlichia chaffeensis reported to the CDC has more than doubled from 2007-2017. E. chaffeensis is the most common cause of Ehrlichiosis [1]. It has previously been reported that around $20 \%$ of infected patients develop neurological manifestations [2]. Neurological symptoms can include confusion, lethargy, photophobia, seizures, headaches, ataxia, hyperreflexia, clonus, delirium, encephalitis, and meningitis. We present what may be the first case series of two patients with Ehrlichia meningoencephalitis along with one suspected case that presented to our institution within a two-month span (Figure 1).

\section{Case Presentation}

\section{Case 1}

A 71-year-old male presented to our institution in June of 2019 with acute encephalopathy and fever. He resided in a wooded area in Southeast Ohio and his sister noticed ticks were on him within the past month leading up to admission. Blood cultures, Chest $\mathrm{X}$-Ray (CXR), and Urinalysis (UA) were all negative. His labs were notable for a mild transaminitis and thrombocytopenia. CT Head and MRI Brain showed no acute findings. On exam, he had nuchal rigidity and was noted to be acutely delirious without any

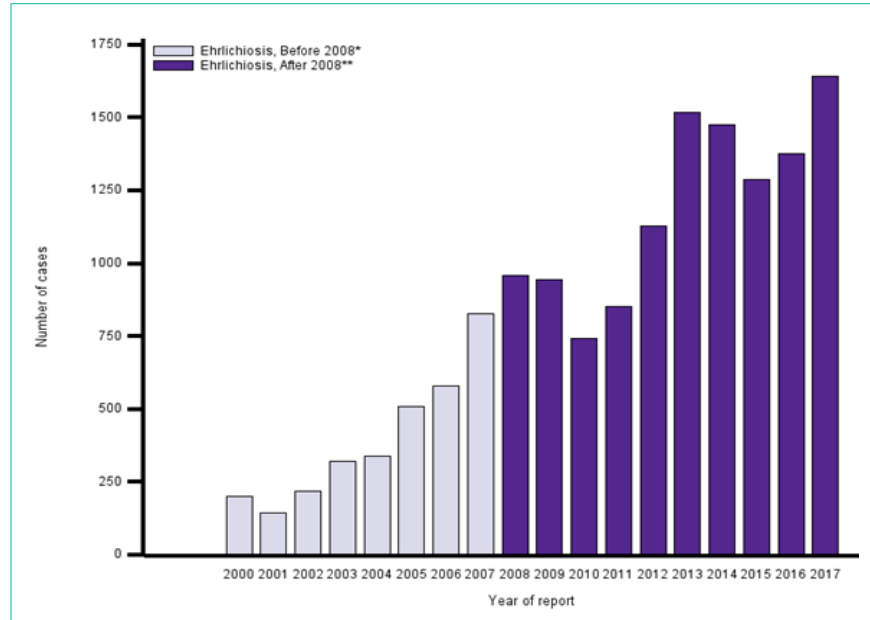

Figure 1: Number of U.S. Ehrlichiosis cases caused by E. chaffeensis and reported to the CDC (2000-2017) (obtained from the CDC).

focal neurological deficits. He was initially started on Vancomycin, Ceftriaxone, Acyclovir, Ampicillin/Sulbactam and Doxycycline. Lumbar Puncture (LP) revealed a lymphocytic pleocytosis. His paraneoplastic and meningitis/encephalitis panel were negative. 


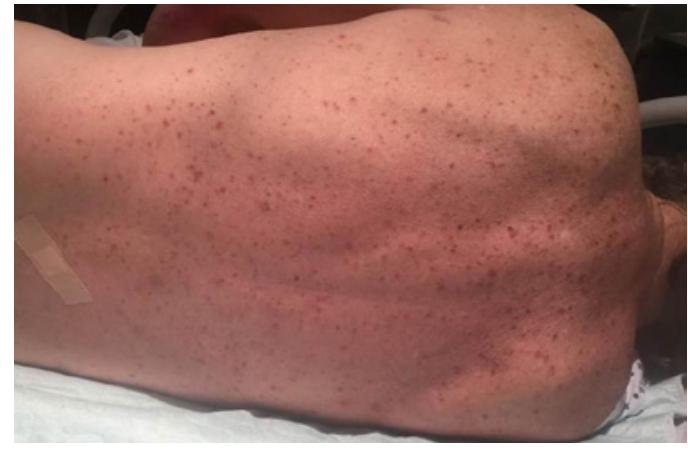

Figure 2: Case 1 had a maculopapular rash classically seen in Ehrlichiosis.

His 14-3-3 antigen was positive and his RT-QUIC was negative in Cerebrospinal Fluid (CSF). An infectious serology sent to the Mayo Clinic revealed he was positive for $E$. chaffeensis. He was continued on Doxycycline 100mg every 12 hours for a 14-day course. His mental status and lab abnormalities gradually improved over an 11-day span after Doxycycline was started. He was discharged to a skilled nursing facility from the hospital. On telephone follow up five months later, his wife stated that he was nearly back to his baseline (Figure 2).

\section{Case 2}

A 67-year-old male presented to an outside hospital in May of 2019 due to acute encephalopathy and headache for the past week. He lived in Southeast Ohio and was reported to be involved in lot of outdoor activity. Family stated three ticks were found on him within a month prior to admission. At the outside hospital, he was febrile and his CT Head and MRI Brain showed no acute abnormalities. Upon transfer to our institution, he was confused, hypotensive, tachycardic and febrile. He was started on Vancomycin, Ceftriaxone, Acyclovir, Ampicillin and Doxycycline. His labs were significant for leukopenia, anemia, thrombocytopenia and transaminitis. His CXR, UA and blood cultures were negative for infection. His LP revealed a neutrophilic pleocytosis with elevated protein. His paraneoplastic and meninigitis/encephalitis panel were negative. His infectious serology was positive for E. chaffeensis. On hospital day two, he had a brief generalized seizure. He was loaded with Levetiracetam and started on maintenance therapy. Long-term video Electroencephalography (EEG) for 24 hours did not reveal epileptiform discharges. His mental status and lab values improved over 15 days. Empiric therapy was stopped on day four and he was continued on Doxycycline 100mg BID for 21 days. He was back to baseline on follow up at five months.

\section{Case 3}

A 71-year-old female presented to an outside hospital in June of 2019 with acute encephalopathy for the past two to three days. She lived with her friend in the countryside of Southeast Ohio. She regularly walked her son's dog in nearby wooded areas. Her son stated that they see a lot of lone star ticks in their area. At the outside hospital, her CT Head had no acute findings. Blood cultures, CXR and UA were negative for infection. Her labs were remarkable for mild transaminitis, leukopenia, thrombocytopenia and an elevated Creatinine Kinase (CK). Upon admission to our institution, she was febrile, hypotensive and tachycardic. Her lactate was elevated at $1.95 \mathrm{mmol} / \mathrm{L}$. She was initially started on Vancomycin, Ceftriaxone,
Table 1: Summary of Case presentations.

\begin{tabular}{|c|c|c|c|}
\hline & Case 1 & Case 2 & Case 3 \\
\hline Age & 71 & 67 & 71 \\
\hline Location & Southeast Ohio & Southeast Ohio & Southeast Ohio \\
\hline Month & June & May & June \\
\hline $\begin{array}{c}\text { Platelets (K/uL) POA } \\
\rightarrow \text { Discharge }\end{array}$ & $66 \rightarrow 159$ & $55 \rightarrow 269$ & $45 \rightarrow 233$ \\
\hline $\begin{array}{c}\text { AST/ALT (U/L) POA } \rightarrow \\
\text { Discharge }\end{array}$ & $105 / 27 \rightarrow 48 / 21$ & $82 / 40 \rightarrow 32 / 32$ & $198 / 97 \rightarrow 43 / 33$ \\
\hline \multirow{3}{*}{ CSF Profile } & $\begin{array}{l}\text { WBC (uL): } 44 \\
\text { Lymphocytes } \\
\text { (\%): } 81\end{array}$ & $\begin{array}{l}\text { WBC (uL): } 7 \\
\text { Lymphocytes } \\
\text { (\%): } 52\end{array}$ & \multirow{3}{*}{$\begin{array}{l}\text { LP unable to be } \\
\text { performed }\end{array}$} \\
\hline & $\begin{array}{l}\text { Neutrophils } \\
\text { (\%): } 1\end{array}$ & $\begin{array}{l}\text { Neutrophils } \\
\text { (\%): } 25\end{array}$ & \\
\hline & $\begin{array}{l}\text { Protein }(\mathrm{mg} / \\
\mathrm{dL}): 79\end{array}$ & $\begin{array}{l}\text { Protein }(\mathrm{mg} / \\
\mathrm{dL}): 86\end{array}$ & \\
\hline $\begin{array}{c}\text { Ehrlichia chaffeensis } \\
\text { PCR }\end{array}$ & + & + & + \\
\hline Doxycycline started & Hospital Day 3 & Hospital Day 2 & Hospital Day 2 \\
\hline Follow up at 5 months & Close to baseline & $\begin{array}{l}\text { Back to } \\
\text { baseline }\end{array}$ & Back to baseline \\
\hline
\end{tabular}
POA: Present on Admission.

Acyclovir, Ampicillin and Doxycycline. Continuous video EEG showed no seizures or epileptiform discharges. MRI Brain showed no acute changes. LP was not obtained due to severe thrombocytopenia. Her infectious serology was positive for E. chaffeensis. She was treated with Doxycycline $100 \mathrm{mg}$ every 12 hours for a 10-day course. Her mental status and lab values improved over six days. On follow up five months later, she stated that she was back to baseline (Table 1).

\section{Discussion}

According to the CDC, Ehrlichiosis became a reportable disease in 1999 and the first reported data was in 2000. The majority of cases reported to the CDC occur during June and July. Cases of Ehrlichia are more commonly reported in men than in women. The highest number of cases occurs in people aged 60-69. The number of cases due to $E$. chaffeensis reported to the $\mathrm{CDC}$ has been steadily increasing since 2000 .

The white-tailed deer is the natural reservoir for E. chaffeensis [3]. Upon vector transmission to humans, Ehrlichia species replicate intracellularly in the reticuloendothelial system. Symptoms are most commonly fever, headache, myalgias and arthralgias and typically manifest after a 5-10 day incubation period [4]. A maculopapular rash that spares the palms and soles can be present in a third of adult patients $[5,6]$. Approximately $20 \%$ of patients develop neurological manifestations [2]. $10 \%$ of cases have displayed signs of meningeal irritation including nuchal rigidity, photophobia and severe headache [7].

E. chaffeensis PCR has high specificity (60-85\%) and sensitivity (60-85\%) and is the confirmatory test of choice. Peripheral blood, bone marrow, or CSF smear analysis looking for morulae is the most specific for Ehrlichiosis diagnosis; however, Wright Giemsa stains are relatively insensitive as only $3 \%$ of patients have monocytes with morulae [12]. Most cases demonstrate a lymphocytic pleocytosis in CSF; however, one-third of cases can have a neutrophilic predominance [14]. The sensitivity of identifying Ehrlichia in CSF through PCR is unknown.

Treatment should be with Doxycycline 100mg twice daily for 
10-14 days. In a 10-year retrospective review of 46 patients with confirmed Ehrlichia or Anaplasma infections, patients who had doxycycline initiated in the first 24 hours were compared to patients who did not have doxycycline empirically. Delay in doxycycline was associated with increased rates of mechanical ventilation and longer hospital stays [15].

A PubMed literature search using the words "Ehrlichiosis and meningoencephalitis" revealed five case reports with CNS manifestations. Presentations included altered mental status, status epilepticus or severe headaches. One patient rapidly deteriorated and died. The others were treated with Doxycycline with good recovery [7-11].

This may be the first case series of Ehrlichia meningoencephalitis and is noteworthy due to the rising prevalence of Ehrlichiosis. In patients who have been exposed to outdoor areas where lone-star ticks reside and who present in the summer months with fever, altered mental status, thrombocytopenia and transaminitis, Ehrlichia meningoencephalitis should be high on the differential diagnosis. All of our patients were aged 67-71. None were immunosuppressed. Neuroimaging findings were nonspecific. CSF studies showed a lymphocytic or neutrophilic pleocytosis with mild protein elevation. PCR technique allows for direct detection of pathogen-specific DNA and is the preferred method of detection during the acute phase of illness. Prompt treatment with Doxycycline $100 \mathrm{mg}$ twice daily led to excellent outcomes in all our patients. Typical meningitis coverage does not include Doxycycline; therefore, it is important to recognize this disease early on. Ehrlichia meningoencephalitis has the potential to be a fatal disease, but high suspicion and good clinical acumen can result in good outcomes for these patients.

\section{Declaration}

All patients gave their informed consent to be included in the study.

All authors contributed to the study conception and designs. Material preparation and analysis were performed by Jonathan Morena, Marika Antimisiaris and Dilip Singh. Jonathan Morena wrote the first draft of the manuscript and all authors commented on previous versions of the manuscript. All authors read and approved the final manuscript.

\section{References}

1. Centers for Disease Control and Prevention. Ehrlichiosis. 2017.

2. Bennett J, Dolin R, Blaser MJ. Mandell, Douglas and Bennett's Principles and Practice of Infectious Diseases. $8^{\text {th }}$ Ed. Ch. 2015; 187: 2194-2197.

3. Salinas LJ, Greenfield RA, Little SE, Voskuhl GW. Tickborne Infections in the Southern United States. The American Journal of Medical Sciences. 2010; 340: 3 .

4. Ismail N, McBride J. Tick-Borne Emerging Infections. Clinics in Laboratory Medicine. 2017; 37: 317-340

5. Biggs H. Diagnosis and Management of Tickborne Rickettsial Diseases: Rocky Mountain spotted fever and Other Spotted Fever Group Rickettsioses, Ehrlichioses and Anaplasmosis-United States. 2016; 65: 1-44.

6. Ratnasamy N, Everett ED, Roland WE, McDonald G, Caldwell CW. Central Nervous System Manifestations of Human Ehrlichiosis. Clinical Infectious Disease. 1996; 23: 314-319.

7. Beavers C, Rickard K, Hollensead SC, Snyder JW. Two fatal Ehrlichia cases with complete autopsies. JMM Case Rep. 2014; 1: 000349.

8. Dredla B, Freeman WD. Ehrlichia meningitis mimicking aneurysma subarachnoid hemorrhage. Neurohospitalist. 2016; 6: 76-79

9. Ugbarugba E. Aseptic meningitis secondary to Ehrlichia chaffeensis. J Hosp Med. 2015; 10.

10. Ahkee S, Ramirez J. A case of concurrent Lyme meningitis with ehrlichiosis. Scand J Infect Dis. 1996; 28: 527-528.

11. Buzzard SL, Bissell BD, Bastin MLT. Ehrlichiosis presenting as severe sepsis and meningoencephalitis in an immunocompetent adult. JMM Case Reports. 2018; 5: e005162.

12. Sathiamoorthi S, Smith W. The eye and tick-borne disease in the United States. Current Opinion Ophthalmology. 2016; 27: 530-537.

13. Seraji-Bozorgzad N, Tselis AC. Non-Lyme Tick-Borne Diseases: A Neurological Perspective. Current Neurology Neuroscience Report. 2013; 13: 388 .

14. Hongo I, Bloch KC. Ehrlichia infection of the central nervous system. Curr Treat Options Neurol. 2006; 8: 179-184.

15. Hamburg BJ, Storch GA, Micek ST, Kollef MH. The importance of early treatment with doxycycline in human ehrlichiosis. Medicine. 2008; 87: 53-60. 